\title{
The Role of Heterogeneities as a Tuning Parameter of Earthquake Dynamics
}

\author{
Gert Zöller, ${ }^{1}$ Matthias Holschneider ${ }^{2}$ and Yehuda Ben-Zion ${ }^{3}$
}

\begin{abstract}
We investigate the influence of spatial heterogeneities on various aspects of brittle failure and seismicity in a model of a large strike-slip fault. The model dynamics is governed by realistic boundary conditions consisting of constant velocity motion of regions around the fault, static/kinetic friction laws, creep with depth-dependent coefficients, and 3-D elastic stress transfer. The dynamic rupture is approximated on a continuous time scale using a finite stress propagation velocity ("quasidynamic model"). The model produces a "brittle-ductile" transition at a depth of about $12.5 \mathrm{~km}$, realistic hypocenter distributions, and other features of seismicity compatible with observations. Previous work suggested that the range of size scales in the distribution of strength-stress heterogeneities acts as a tuning parameter of the dynamics. Here we test this hypothesis by performing a systematic parameter-space study with different forms of heterogeneities. In particular, we analyze spatial heterogeneities that can be tuned by a single parameter in two distributions: (1) high stress drop barriers in near-vertical directions and (2) spatial heterogeneities with fractal properties and variable fractal dimension. The results indicate that the first form of heterogeneities provides an effective means of tuning the behavior while the second does not. In relatively homogeneous cases, the fault self-organizes to large-scale patches and big events are associated with inward failure of individual patches and sequential failures of different patches. The frequency-size event statistics in such cases are compatible with the characteristic earthquake distribution and large events are quasi-periodic in time. In strongly heterogeneous or near-critical cases, the rupture histories are highly discontinuous and consist of complex migration patterns of slip on the fault. In such cases, the frequency-size and temporal statistics follow approximately power-law relations.
\end{abstract}

Key words: Earthquake dynamics, fault models, seismicity, ruptures, heterogeneities, criticality.

\section{Introduction}

Theoretical parameter-space studies of earthquake models aim to provide a physical basis for understanding seismicity. Such studies complement observational efforts to collect better and larger data sets, and statistical efforts to establish better estimation procedures. In this context, the main goal of the fault model studies is to

\footnotetext{
${ }^{1}$ Institute of Physics and Institute of Mathematics, University of Potsdam, Potsdam, Germany (e-mail: gert@agnld.uni-potsdam.de)

${ }^{2}$ Institute of Mathematics, University of Potsdam, Potsdam, Germany; on leave from CNRS Rennes, France (e-mail: hols@math.uni-potsdam.de)

${ }^{3}$ Department of Earth Sciences, University of Southern California, Los Angeles, USA (e-mail: benzion@usc.edu)
} 
identify different physical regimes that may be present in the data. This can provide guidelines for separating the observations to different corresponding populations, thereby allowing higher-resolution (i.e., more space-time specific) information to be extracted from the available data. In this work we attempt to provide a better understanding of the role of heterogeneities on earthquake dynamics. Toward this goal we first develop a description of heterogeneities that is likely to be physically relevant and has a small number of parameters. We then perform a systematic parameter-space study using that form of heterogeneities and a model of a segmented strike-slip fault in elastic half space.

Important unresolved questions are the dependencies of the frequency-size and temporal statistics of earthquakes on the physical properties and parameters of the fault. The most general feature of seismicity is probably the Gutenberg-Richter (GR) law (Gutenberg and Richter, 1956) for the frequency of earthquake occurrence, $\log N=a-b m$, where $a$ and $b$ are constants and $N$ is the number of earthquakes with magnitudes greater than or equal to $m$. When phrased in terms of seismic potency (average slip times rupture area) or moment (rigidity times potency), the GR relation follows a power-law distribution (e.g., TURCOTTE, 1997; BEN-ZION, 2003). It has been observed that the GR law is valid over a broad range of magnitudes for data collected in large regions of space (e.g., UTSU, 2002). However, on individual fault zones the situation is different: although power-law behavior is in general present, certain ranges of earthquake magnitudes are characterized by clear deviations from power-law scaling. Observed frequency-size statistics on individual faults are found to exist between two end-member cases: (1) a Gutenberg-Richter law with a fall-off for large magnitudes, and (2) a power law for small earthquakes followed by a gap for intermediate earthquakes and a peak for frequently-occurring characteristic events.

Several explanations for the occurrence of the different frequency-size distributions have been derived from fault models. Here it is crucial to distinguish whether or not an earthquake can rupture large parts of the fault (critical or supercritical behavior), or is stopped after short propagation due to spatial barriers or stress loss during rupture (subcritical behavior). BEN-ZION and RICE (1993, 1995) and BENZION (1996) examined this issue in terms of stress concentration and strength heterogeneities in elastic solids. Lomnitz-AdLer (1999), STEACY and Mccloskey (1999) and others studied the same with cellular automata models. HAINZL and ZÖLLER (2001) studied this question in terms of stress concentration and spatial disorder in a spring-block model. FISHER et al. (1997) and DAHMEN et al. (1998) have shown that coseismic dynamic weakening and stress loss in a mean field version of the elastic half-space model of BEN-ZION and RICE (1993) can produce the different end-member frequency-size statistics and spontaneous transitions between them. ZÖLLER et al. (2004) generalized the phase diagram of DAHMEN et al. (1998) to a more realistic model with (non-mean field) elastic Green's function for the coseismic stress transfer and several other features (e.g., gradual strength healing and causal propagation of stress). 
It is widely accepted that spatial disorder, or heterogeneities, significantly influence rupture propagation and thus the frequency-size statistics and other aspects of earthquake behavior. Such heterogeneities may have various forms. The locations of large asperities (e.g., the Parkfield asperity) are known from seismological and other studies (Wyss et al., 2000) and can be implemented easily in fault models. In contrast, small-scale heterogeneities are in general unknown with respect to their sizes, forms and positions. Therefore, heterogeneities in model simulations are often based on simple assumptions, e.g., uniformly distributed random numbers with a certain range, or power-law distributions characterized by range of values and fractal dimensions. Both types of heterogeneities produce stress fluctuations that may be associated with smoothness or roughness of the fault. The results of such studies indicate that large (characteristic) earthquakes can evolve on smooth faults, whereas the growth of a rupture is terminated earlier on a rough fault. A natural example for a smooth fault is the San Andreas fault, which is characterized by a GutenbergRichter law for small events and quasiperiodic occurring "characteristic" large events. In contrast, the San Jacinto fault with its numerous offsets and branches has a highly irregular geometry and is governed by Gutenberg-Richter distributed seismicity over the entire range of magnitudes (WESNOUSKY 1994; STIRLING et al., 1996). In this paper, we examine whether the degree of spatial heterogeneity can be tuned by one or two parameters in a fault model between these two extreme cases. This would allow a description of the heterogeneity in terms of the phase diagram approach of DAHMEN et al. (1998) and ZÖLLER et al. (2004).

BEN-ZION and RICE (1993, 1995, 1997) studied evolutionary seismicity on homogeneous and heterogeneous fault systems using several types of calculations ranging from quasi-static to fully-dynamic. The results from these and related analytical and numerical studies (BEN-ZION et al., 1999; DAHMEN et al., 1998; BENZION, 2001; FisHeR et al., 1997; ZÖLLER et al., 2004; MEHTA et al., 2005) indicate that the essential long-term properties of earthquake statistics, and overall aspects of additional dynamic quantities such as moment-rate functions and slip distributions, remain the same when the models share certain key features. These include the physical dimension, range of interaction, overall class of heterogeneities (e.g., continuum vs. inherently discrete cases), and the existence of dynamic weakening and dissipation. Such models are referred to as belonging to the same universality class and they share the same coarse-grained properties that govern the behavior on large space-time scales (e.g.,WILSON 1979; BINNEY et al., 1993).

The goal of the present work is to study phenomena associated with large-scale ruptures in realistic heterogeneous fault structures. As discussed by BEN-ZION and RICE (1993, 1995, 1996), the successful propagation of rupture in such systems involves stresses that operate at finite distances from the rupture front, where slip can nucleate at non-contiguous locations across fault offsets and other strong barriers. Thus the large-scale growth of slip instabilities in realistic segmented fault systems is governed by length scales that are much larger than those associated with crack-tip 
processes such as strength degradation with slip. We therefore neglect detailed smallscale crack-tip processes and fully dynamic calculations, and focus on large-scale fault interactions given approximately by macroscopic static/kinetic friction and creep property values (BEN-ZION, 1996). The assumed properties are assigned to a system of numerical cells representing quasi-independent fault segments, each behaving uniformly in the adopted modeling approximation. Our investigations are done with quasi-dynamic calculations (ZöLLER et al., 2004) that incorporate causal spatio-temporal propagation of stress during an earthquake failure. This allows us to discuss basic differences of rupture histories on smooth and rough faults.

Power-law distribution of fault properties may be used to tune the smoothness of a fault by two parameters, the fractal dimension $D$ and standard deviation of values. It is, however, unlikely that the active structure of large faults or fault systems is characterized by fractal properties over tens or hundreds of kilometers (BEN-ZION and SAmmis, 2003). Instead, it is more realistic to use heterogeneities that model large-scale offsets and branches superimposed on a relatively smooth fault. BEN-ZION and RICE (1995) and BEN-ZION (1996) used such a form of heterogeneities consisting of strong (high stress drop) barriers separating weaker (low stress drop) fault segments. To model realistic structures, the barriers were assumed to be strongly correlated with depth and essentially uncorrelated in the strike direction. In the following sections we perform a systematic study of frequency-size statistics and other aspects of earthquake dynamics using such nearly-vertical barriers embedded in an overall smooth fault, as well as power-law distributions, and discuss the results in terms of the phase diagram approach.

\section{Model Framework}

Our model includes a single rectangular fault embedded in a 3-D elastic half space (Fig. 1). A fault region of $70 \mathrm{~km}$ length and $17.5 \mathrm{~km}$ depth is covered by a computational grid, divided into $128 \times 32$ uniform cells, where deformational processes are calculated. Tectonic loading is imposed by a motion with constant velocity $v_{\mathrm{pl}}=35 \mathrm{~mm} /$ year of the regions around the computational grid. The space-dependent loading rate provides realistic boundary conditions. Using the static stress transfer function $K\left(x, z ; x^{\prime}, z^{\prime}\right)$ from CHINNERY (1963), the continuous tectonic loading for each cell $(x, z)$ on the computational grid is a linear function of time $t$ and plate velocity $v_{\mathrm{pl}}$ :

$$
\Delta \tau(x, z ; t)=\left(-v_{\mathrm{pl}} \cdot t\right) \cdot \sum_{x^{\prime}, z^{\prime} \in \text { grid }} K\left(x, z ; x^{\prime}, z^{\prime}\right),
$$

where the minus sign stems from the fact that forward (right-lateral) slip of regions around a locked fault segment is equivalent to back (left-lateral) slip of the locked fault segment. Additional loadings on a given cell occur due to brittle and creep failures on the fault. 


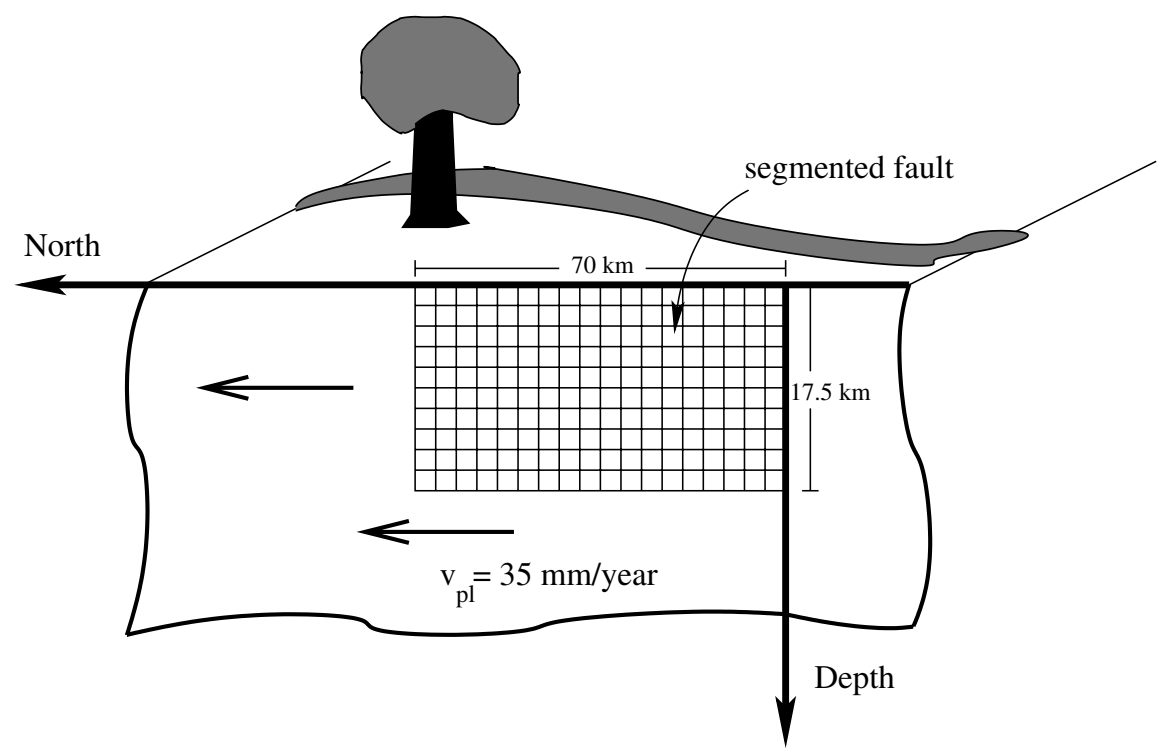

Figure 1

A sketch of a 2-D strike-slip fault in a 3-D elastic half space.

While the loadings produce an increase of stress on the fault, the local stress may be reduced by creep and brittle failure processes operating "in series."

\subsection{The Creep Process}

The ongoing creep motion on the fault is implemented as in BEN-ZION (1996). The space- and time-dependent creep rate follows the formula

$$
\dot{u}_{\text {creep }}(x, z, t)=c(x, z) \tau(x, z, t)^{3}
$$

where $c(x, z)$ are time-independent coefficients and $\tau$ is the local stress.

Equation (2) corresponds to dislocation creep observed in laboratory experiments with a coefficient $c$ that increases with temperature and pressure. Following BENZION (1996), we choose a distribution $c(x, z)$ that simulates "brittle-ductile" transitions in the vertical and horizontal directions:

$$
c(x, z)=A \exp \left(B \cdot \operatorname{Max}\left(\left(x-x_{D B}\right),\left(z-z_{D B}\right)\right)+\operatorname{ran}(x, z),\right.
$$

where $A$ and $B$ are constants and $x_{B D}=62.5 \mathrm{~km}, z_{B D}=10.0 \mathrm{~km}$ are the horizontal and vertical positions of the "brittle-ductile" transition zones. The transition in depth models the occurrence of ductile deformation with increasing temperature and pressure, while the transition along strike is based on the assumption that an essentially brittle fault segment is connected with a creeping fault section. At $x_{B D}$ and $z_{B D}$, the creep rates equal approximately the tectonic loading rate, the stress increase 
due to the plate motion is released mostly by aseismic creep, and consequently hypocenters do not nucleate. The values $\operatorname{ran}(x, z)$ simulate a certain mixing between the two zones which are dominated by different brittle and ductile properties. Details about the calculation of $A$ and $B$ and the values of $\operatorname{ran}(x, z)$ are given in BEN-ZION (1996). We point out that this choice of creep coefficients represents a certain type of quenched spatial heterogeneities, which are, however, constant in all simulations. The gradual creep motion results in realistic depth profiles of the simulated hypocenter distribution, which follow approximately a Gaussian function in agreement with observations.

Taking into account the relation between stress and displacement on the computational grid

$$
\Delta \tau(x, z)=\sum_{x^{\prime}, z^{\prime} \in \text { grid }} K\left(x, z, x^{\prime}, z^{\prime}\right) \Delta u\left(x^{\prime}, z^{\prime}\right) .
$$

Equations (2)-(4) result in a system of $128 \times 32$ coupled ordinary differential equations, which is solved numerically using a Runge-Kutta scheme. However, in order to increase numerical efficiency we use the decay of the elastic Green's function to reduce the interaction during the creep to a local neighborhood.

\subsection{The Brittle Process}

The brittle process is governed by a static/kinetic friction law and dynamic overshoot. A brittle failure is initiated on a cell if the static friction $\tau_{s}$ is exceeded. We assume that the static threshold increases with depth $z$ :

$$
\tau_{s}(z)=\Delta \tau_{\max }+f \cdot z,
$$

where $f=13.5 \mathrm{MPa} / \mathrm{km}$ and $\Delta \tau_{\max }$ is the maximum allowed brittle stress drop (BENZION, 1996). If a cell slips, the stress drops to an arrest stress $\tau_{a}=\tau_{s}-\Delta \tau$, where the stress drop $\Delta \tau$ is the parameter that defines the heterogeneity, e.g., $\Delta \tau$ can be drawn from a power-law distribution or from a set of two values representing high stress drop and low stress drop regions.

Following an initial failure during an earthquake, the brittle threshold drops from the static value $\tau_{s}$ to a dynamic value $\tau_{d}$, which is calculated in relation to a dynamic overshoot coefficient DOS:

$$
\tau_{d}=\tau_{s}-\frac{\tau_{s}-\tau_{a}}{D O S} .
$$

In our study, we use the value $D O S=1.25$ for the dynamic overshoot (BEN-ZION and Rice, 1993; Madariaga, 1976).

An important aspect of the brittle process is the dynamic weakening coefficient $\varepsilon$ :

$$
\varepsilon=\frac{\tau_{s}-\tau_{d}}{\tau_{s}},
$$


which becomes space-dependent if $\Delta \tau$ is a function of space and DOS is a constant number.

The coseismic stress transfer is calculated by means of the three-dimensional solution of CHINNERY (1963) for static dislocations on rectangular patches in an elastic Poisson solid with rigidity $\mu=30 \mathrm{GPa}$. In particular, we approximate the $3+1$ dimensional space-time stress transfer by

$$
\Delta \tau(x, z ; t)=(1-\gamma) \cdot \sum_{\left(x^{\prime}, z^{\prime}\right) \in \text { grid }} K\left(x, z ; x^{\prime}, z^{\prime}\right) \Delta u\left(x^{\prime}, z^{\prime} ; t-r / v_{s}\right),
$$

where $\Delta u$ is the slip, $r$ is the spatial distance between the cells $(x, z)$ and $\left(x^{\prime}, z^{\prime}\right)$, and $v_{s}$ is the shear wave velocity.

The factor $(1-\gamma)$ is in the range $(0 ; 1]$ and corresponds to a given ratio of rigidities governing during instabilities the self-stiffness of a slipping cell (diagonal elements of the stiffness matrix $K$ ) and the stress transfer to the surrounding domain (off-diagonal elements). A ratio smaller than 1 represents stress loss during rapid slip on the fault to internal free surfaces in the solid associated with porosity and cracks. We refer to $\gamma$ and $1-\gamma$ as the stress loss parameter and stress conservation parameter, respectively. FISHER et al. (1997) and DAHMEN et al. (1998) showed analytically that at $\varepsilon=0$ and $\gamma=0$ the model has a critical point of phase transition.

The slip $\Delta u(x, z)$ of a cell at position $(x, z)$ is related to the stress drop $\Delta \tau(x, z)$ at the same position through the self-stiffness: $\Delta u(x, z)=\Delta \tau(x, z) / K(x, z ; x, z)$. The size of an earthquake is measured by the potency (e.g., BEN-ZION, 2003), which is the integral of the slip $\Delta u$ over the rupture area $A$,

$$
P=\int_{A} \Delta u(x, z) d x d z
$$

We note that Equation (8) corresponds to the quasi-dynamic model of ZöLLER et al. (2004), which reduces for $\gamma=0$ and $v_{s} \rightarrow \infty$ to the quasi-static model of BEN-ZION (1996). The quasi-dynamic model has the advantage that the spatio-temporal evolution of stress transfer during an earthquake can be analyzed, whereas the rupture in the quasi-static model occurs instantaneously. This allows us to study rupture properties in model realizations characterized by different degrees of heterogeneity. Similar quasi-dynamic procedures were used by RoBINSON and Benites (2001) and Heimpel (2003).

\subsection{Heterogeneities}

In this study we consider two different types of heterogeneities that can be tuned with a single parameter: (1) near-vertical barriers of high stress drop embedded in regions of low stress drop, and (2) continuous power-law distributions of the stress drop with a fixed standard deviation. 
The first type of heterogeneities, hereinafter referred to as RW, represents nearvertical offsets that produce, as discussed by BEN-ZION and RICE (1995) and BENZION (1996), large-scale fault segmentation. To generate the stress drop distribution, we fix randomly a certain number $k$ of cells along the free surface that act as initiators of the barriers. The continuation of the barriers with depth is determined by a quasi2-D random walk, where with each depth increment the barrier may move one unit to the left or right. Using different values of $k$ results in different degrees of complexity, e.g., for $k=1$ two continuous patches with low brittle stress drop are separated by one high stress drop barrier representing an overall relatively smooth fault. The roughness or segmentation of the fault increases if more barriers are added. The degree of heterogeneity is quantified by means of a tuning parameter $h$ which is calculated in the following way: Each cell with a low stress drop is surrounded by the circle of maximum area $A_{i}$ which includes only cells with low stress drop. The circle size $\sqrt{A_{i}}$ is a measure for the distance to the nearest high stress drop barrier or to a fault boundary. The standard deviation of the distribution of the circle sizes gives a measure for the range of size scales. To remove boundary effects, we define the heterogeneity parameter by

$$
h=\operatorname{std}\left(\sqrt{\left|A_{i}-A_{i}^{0}\right|}\right),
$$

where $A_{i}^{0}$ is the corresponding circle area on an "empty" fault (fault without barriers). The dimension of $h$ is $\mathrm{km}$. For a fixed fault, $h$ may be transformed to a dimensionless value, $h_{n}=h / h_{\max }$.

We point out that $h$ is determined only by the distribution of the stress drops $\Delta \tau$. The stress drop values themself are fixed for all simulations of model RW: $\Delta \tau_{h}+R$ is the high stress drop value, $\Delta \tau_{l}+R$ denotes the low stress drop value. $\Delta \tau_{h, l}$ are constant numbers, while $R=\sigma \cdot r n$ is additive noise with a width $\sigma$ and a random number $r n$ in the range $[0 ; 1]$.

For finite faults, $h$ always has a positive value even if no barrier is present. BENZION and RICE (1995) and BEN-ZION (1996) pointed out that the simulated earthquake dynamics depend on the range of size scales of the heterogeneities, and distinguished between wide range of size scales (WROSS) and narrow range of size scales (NROSS). In terms of this concept, $h$ tunes range of size scales represented by the range of radii of the circles discussed above. Specifically, small values of $h$ correspond to NROSS and large values correspond to WROSS.

For the fractal heterogeneities $(\mathrm{F})$, we generate a power-law distribution (see e.g., TurcotTE, 1997) of stress drops between $\Delta \tau_{\min }$ and $\Delta \tau_{\max }$ with a given fractal dimension $D$. Although the fractal dimension allows some tuning of the range of size scales in the distribution of stress drops, model $\mathrm{F}$ belongs is general to the class associated with NROSS (BEN-ZION, 1996). This is because the power-law distribution has a continuous range of stress drop values leading to an overall higher degree of smoothness. In particular, despite the presence of heterogeneities with a range of size 
scales, a rupture can easily overcome such heterogeneities since the stress drops fluctuations at the cell boundaries are relatively small. This is in contrast to model RW, which has a bimodal distribution of stress drop ("high" and "low") with high fluctuations at the barrier boundaries. Model F can be somewhat tuned towards WROSS by increasing the standard deviation of the stress drop distribution. As will be shown, however, we find that power-law distributions do not provide an effective way of tuning the heterogeneities.

\section{Model Simulations}

In the first part of this section, we discuss frequency-size and temporal distributions of seismicity in simulated earthquake catalogs. In the second part, we present the main characteristics of individual ruptures for different ranges of model parameters.

\subsection{Analysis of Earthquake Catalogs}

We generate heterogeneities for the quasi-dynamic model as described in Section 3. The first type of heterogeneities consists of near-vertical high stress drop barriers embedded in a fault with low stress drops. The brittle stress drop in the barriers is $\Delta \tau_{h}=100 \pm 5 \cdot r n$ and the stress drop in the other cells is $\Delta \tau_{l}=10 \pm 5 \cdot r n$, where $r n$ is a uniformly distributed random number between 0 and 1 . The degree of complexity is essentially controlled by the density of barriers. We point out that the total number of points with high/low stress drops does not effect the form of the frequency-size distribution. If this number is changed, the average stress on the fault and inter-patch distances will be modified resulting in a rescaling of the time and magnitude axes. The form of the frequency-size distribution depends only on the distribution of the different brittle properties. Three different RW type distributions of stress drops with different values of $h$ from Eq. (10) are used in our study (Fig. 2). A comparison of the distributions in Figures 2(a) to (c) suggests that $h$ may provide an effective measure for the complexity of the distribution.

The second type of heterogeneities is based on a power-law distribution of brittle stress drops; three employed examples are given in Figure 3. Although the distributions represent smooth and rough surfaces like the RW heterogeneities, the power-law distributions are structurally different: while the RW heterogeneities are characterized by strong localized stress drop gradients which produce high stress fluctuations at these positions on the fault, the power-law distributions are smoother even for high fractal dimensions.

Figure 4(a) shows the cumulative frequency-size distribution for a purely elastic model without stress loss $(\gamma=0)$ and the three stress drop distributions of Figure 2. The results indicate that $h$ acts as a tuning parameter for the frequency-size 

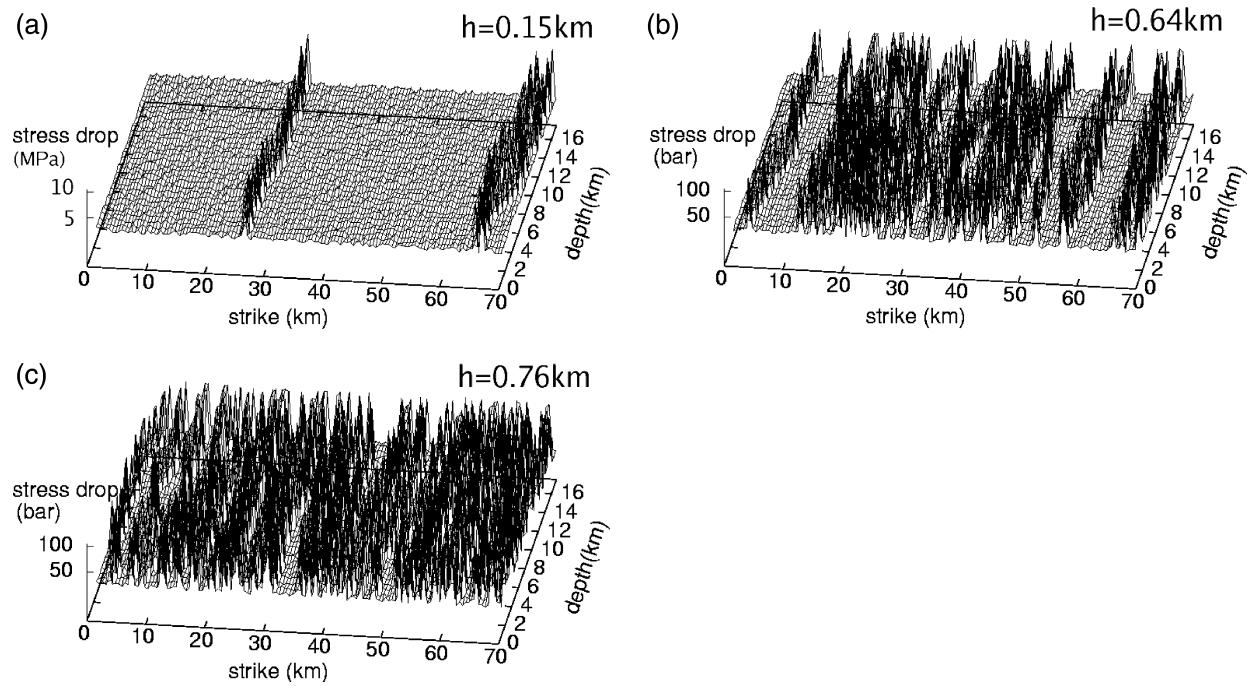

Figure 2

Heterogeneities of the quasi-2D random walk type (RW): The distribution of brittle stress drops consists of high stress drop barriers in a near-vertical direction embedded in a low stress drop environment. The tuning parameter $h$ (Eq. (10)) measures the degree of heterogeneity in units of $\mathrm{km}$.
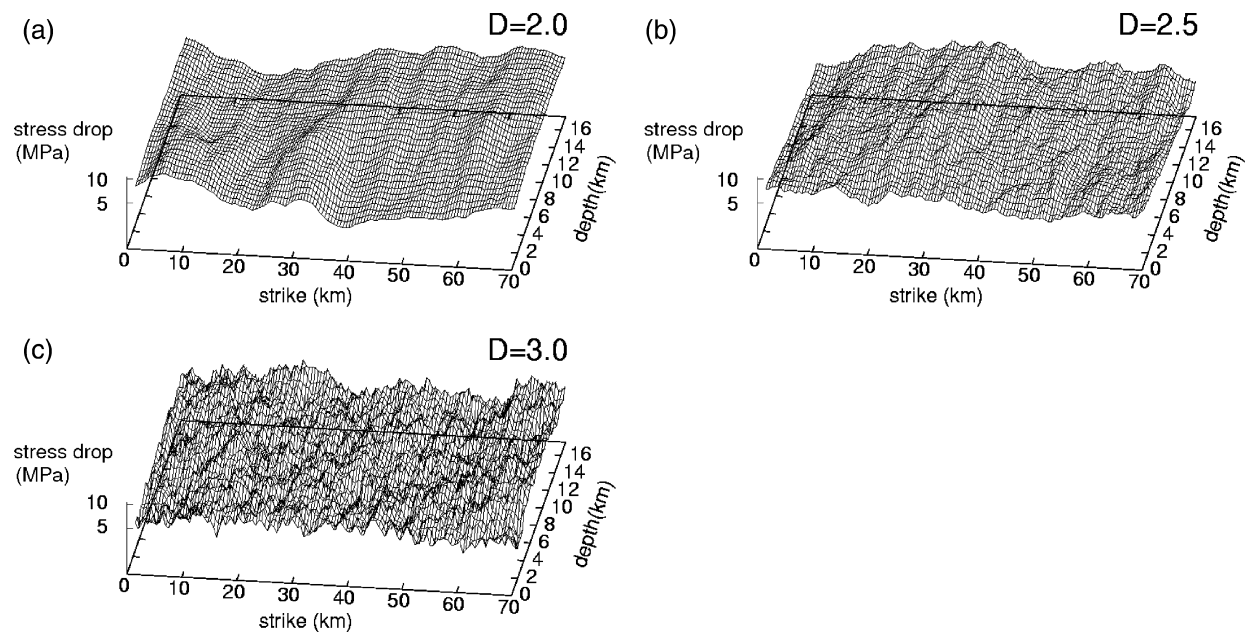

Figure 3

Power-law distributions of the brittle stress drops with three fractal dimensions $D$ and values between 1 $\mathrm{MPa}$ and $9 \mathrm{MPa}$.

distribution. If $h$ is small, the fault is characterized by a NROSS and a few low stress drop patches with relatively uniform size. Only a few ruptures are effected by the high stress drop barriers leading to a characteristic earthquake behavior. In contrast, high 

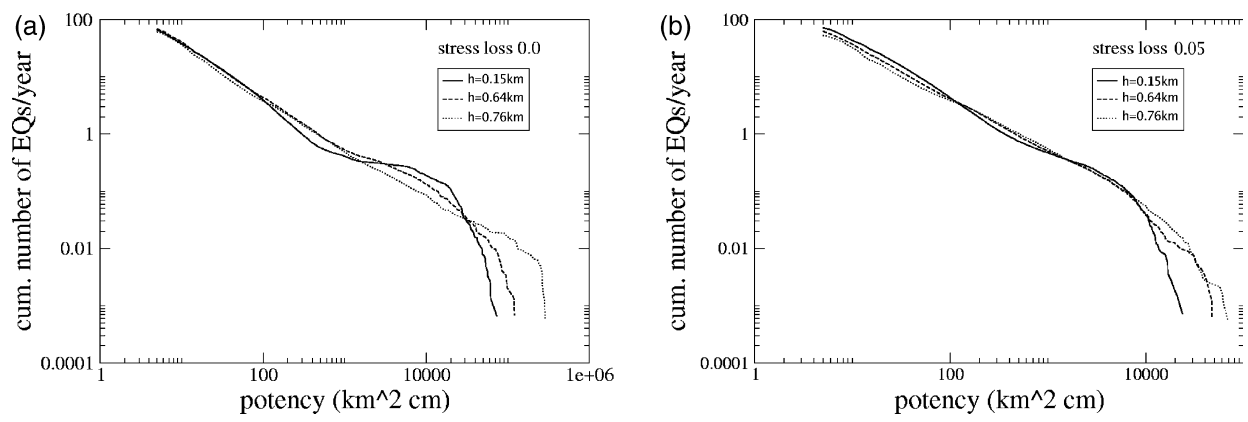

Figure 4

Cumulative frequency-size distributions for RW heterogeneities with different values of $h$ (Eq. (10)) given in Figure 2.

$h$-values representing many low stress drop patches on a fault which has a WROSS, lead to a Gutenberg-Richter distribution with a broader region of power-law scaling. If the number of barriers is increased further, the frequency-size distribution will again show a tendency towards characteristic earthquake behavior, because the barriers will begin to form continuous patches. Consequently, the roles of barriers and surrounding environment will be exchanged and the fault will consist of low stress drop barriers in a high stress drop environment. For physical cases with a finite size, the parameter $h$ can only be considered in certain parts of the interval $\left[0 ; h_{\max }\right]$. The fault with the dimensions shown in Figure 1 and $128 \times 32$ cells has a value of $h_{\max } \approx 0.76 \mathrm{~km}$, while for a fault with $256 \times 32$ cells and $140 \mathrm{~km} \times 17.5 \mathrm{~km}$ $h_{\max } \approx 0.84 \mathrm{~km}$. The frequency-size distribution for the latter case (Figure 5) is qualitatively similar to the distribution of the smaller fault, with the difference that a broader range of heterogeneities is covered. The results for $h=0.84 \mathrm{~km}$ show powerlaw statistics over the entire range of event sizes.

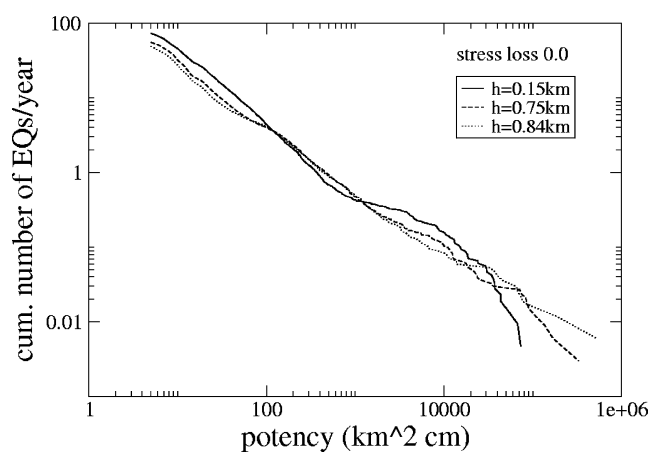

Figure 5

Cumulative frequency-size distributions for RW heterogeneities with different degrees of disorder on a large fault $(256 \times 32$ cells corresponding to a fault of $140 \mathrm{~km}$ length and $17.5 \mathrm{~km}$ depth$)$. 
If a stress loss $\gamma>0$ is introduced, the frequency-size distributions for different values of $h$ become more similar to each other (Fig. 4(b)) in agreement with previous observations of DAHMEN et al. (1998) and ZöLLER et al. (2004).

The frequency-size distributions for the different cases with fractal dimensions and $\gamma=0$ are shown in Figure 6(a). It is seen that the fractal dimension does not provide an effective tuning parameter for the frequency-size distribution. The effect of positive $\gamma$ is again to make the frequency-size distributions more GutenbergRichter like and more similar to each other (Fig. 6(b)).

Figures 7(a) and 7(b) show earthquake sequences (rupture area vs. time) for two realizations of model RW, while Figures 7(c) and 7(d) give similar results for model F. The time series suggest that model RW allows a greater variability of the temporal behavior in terms of the tuning parameter $h$. The results for model $\mathrm{F}$ show an increased occurrence rate for earthquakes with area $A \approx 700$ cells. We note again that model $\mathrm{F}$ represents a smoother fault than model $\mathrm{RW}$, and it has a continuous distribution of stress drops in the range $1 \mathrm{MPa} \leq \Delta \tau \leq 9 \mathrm{MPa}$. This leads to largescale patches which have their own "characteristic events."

The clustering properties of the simulation results can be quantified with the ratio $\mu / \sigma$ of interevent times between large events, where $\mu$ and $\sigma$ are the mean value and standard deviation, respectively. For clustered seismicity $\mu / \sigma<1$, whereas for quasiperiodic occurrence of events $\mu / \sigma>1$. The case $\mu / \sigma=1$ corresponds to a Poisson process. Table 1 shows $\mu / \sigma$ values for the $N$ largest earthquakes in models RW and $\mathrm{F}$ with $\gamma=0$ and different values of the degree of spatial disorder measured by $h$ and $D$. Each value of $N$ corresponds to a magnitude condition $M \geq M_{\text {cut }}$, where $M_{\text {cut }}$ varies slightly for different values of $h$ and $D$. The approximate value of $M_{\text {cut }}$ is also given in the table. The relation between potency $P$ and magnitude $M$ is given by $M=(2 / 3) \log P+3.6 \quad$ (BEN-ZION and RiCE, 1993). For $N=100\left(M_{\text {cut }} \approx 6.5\right)$ and $N=1000\left(M_{\text {cut }} \approx 5.6\right)$, model RW shows a decrease of $\mu / \sigma$ with increasing $h$. In other
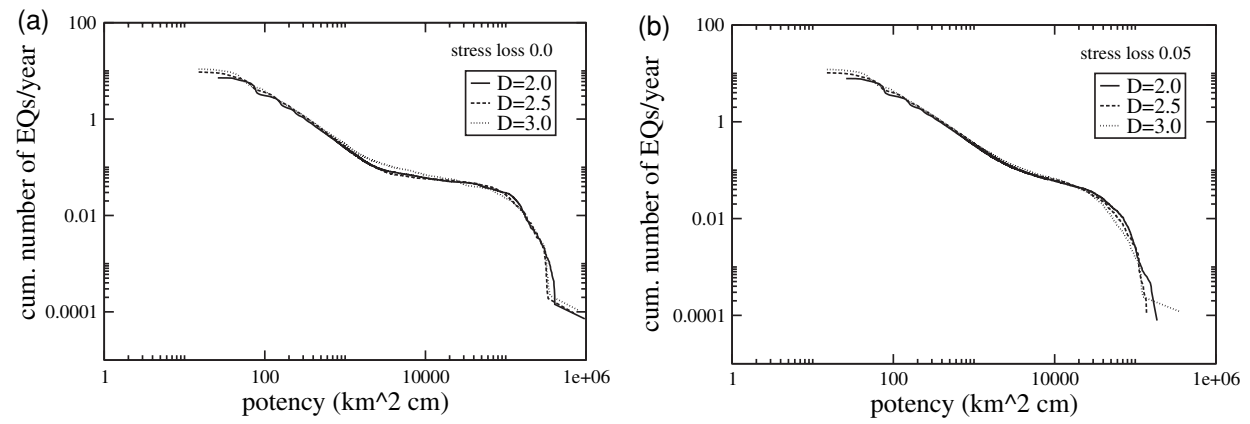

Figure 6

Cumulative frequency-size distributions for fractal heterogeneities with different fractal dimensions $D$ given in Figure 3. 
(a)
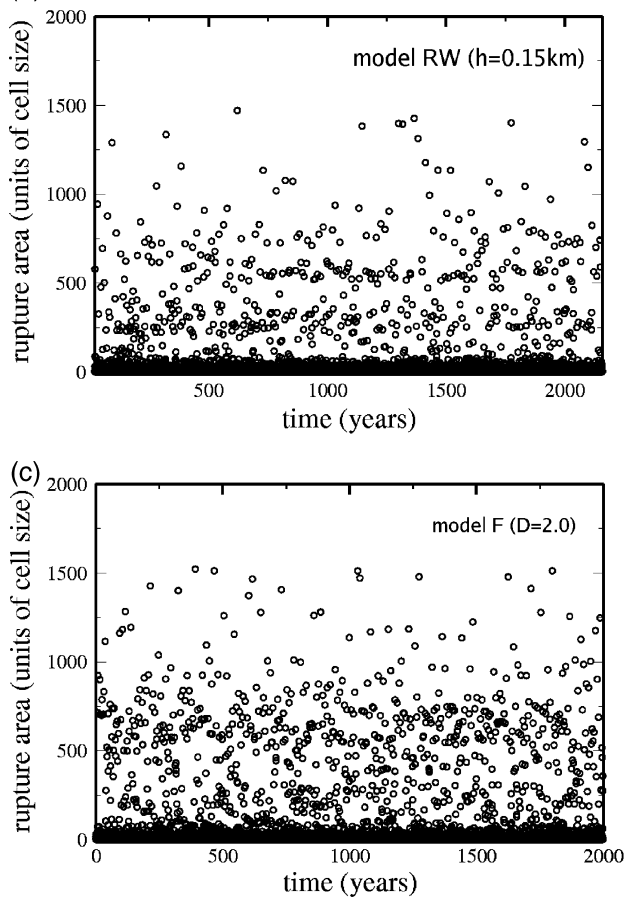

(b)
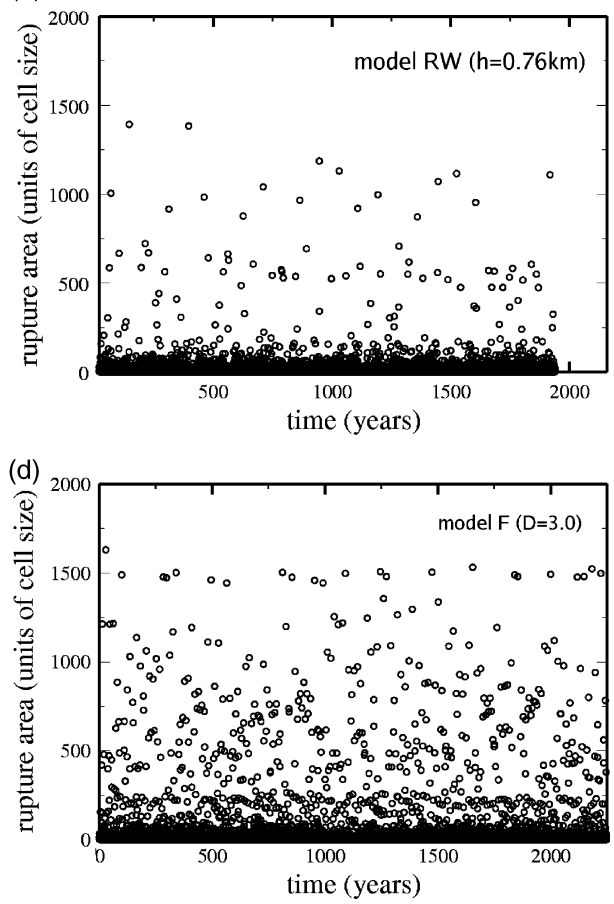

Figure 7

Earthquake sequences (rupture area vs. time) for four simulations with $10^{6}$ events. Panels (a) and (b) correspond to model RW (Figs. 2(a) and (c)); panels (c) and (d) correspond to model F (Figs. 3(a) and (c)).

words, for cases with RW heterogeneities and increasing range of size scales, the temporal behavior changes from a quasi-periodic occurrence of large events toward a random/clustered behavior. For smaller values of $M_{\text {cut }}, \mu / \sigma$ is almost constant and corresponds to random/clustered statistics. This result is supported by the values of $\mu / \sigma$ for the highest degree of disorder $(h=0.84 \mathrm{~km})$ on the large fault $(140 \mathrm{~km} \times 17.5$ $\mathrm{km}$ ), which are also given in Tab. 1. In contrast, model $\mathrm{F}$ has no systematic dependence of $\mu / \sigma$ on the fractal dimension $D$. These observations support our assertion that the distribution type RW is more efficient for tuning the dynamics with a single heterogeneity parameter.

ZÖLLER et al. (2004) have shown that high stress fluctuations occurring along cell boundaries on a heterogeneous fault can cause a spontaneous mode-switching between a Gutenberg-Richter and a characteristic earthquake distribution (DAHMEN et al., 1998; Ben-Zion et al., 1999; WeAtherley et al., 2002). Although the type of distribution does not change in the simulations leading to Figure 7, the results (especially those for model RW) have time periods with clearly different maximum magnitudes. This has a similar origin to the mode-switching: strong stress fluctuations sometimes lead to a spontaneous synchronization or desynchronization 
Table 1

The ratio $\mu / \sigma$ for different model realizations with $\gamma=0$, where $\mu$ is the mean value of interevent times calculated for earthquakes with magnitudes $M \geq M_{\text {cut }}$ and $\sigma$ is the corresponding standard deviation. For statistical reasons, a fixed number $N$ of earthquakes with $M \geq M_{\text {cut }}$ is used. The approximate value of $M_{\mathrm{cut}}$ corresponding to $N$ is given in parentheses. The case $R W L$ represents the large fault $(140 \mathrm{~km} \times 17.5 \mathrm{~km})$ with the highest degree of disorder. In this case, the catalog contains only 16,000 events. Therefore, the value in the last line is given in parentheses

\begin{tabular}{|c|c|c|c|c|c|c|c|}
\hline \multirow{2}{*}{$\begin{array}{c}\text { model } \\
\qquad N\end{array}$} & \multicolumn{3}{|c|}{ RW } & \multicolumn{2}{|l|}{ RWL } & \multicolumn{2}{|c|}{$\mathrm{F}$} \\
\hline & $h=0.15 \mathrm{~km}$ & $h=0.64 \mathrm{~km}$ & $h=0.76 \mathrm{~km}$ & $h=0.84 \mathrm{~km}$ & $D=2.0$ & $D=2.5$ & $D=3.0$ \\
\hline $100\left(M_{\mathrm{cut}} \approx 6.5\right)$ & 1.664 & 1.566 & 1.402 & 1.200 & 1.508 & 1.232 & 1.845 \\
\hline $1000\left(M_{\mathrm{cut}} \approx 5.6\right)$ & 1.480 & 1.180 & 0.990 & 1.005 & 0.869 & 0.790 & 0.826 \\
\hline $10000\left(M_{\mathrm{cut}} \approx 4.9\right)$ & 0.907 & 0.989 & 0.968 & 0.948 & 0.883 & 0.832 & 0.911 \\
\hline $100000\left(M_{\text {cut }} \approx 4.0\right)$ & 0.960 & 0.970 & 0.969 & $(0.938)$ & 0.882 & 0.835 & 0.911 \\
\hline
\end{tabular}

of a large number of cells resulting in an increase or decrease of the maximum magnitude.

The observations discussed above indicate that the relevant parameter of the heterogeneities is the range of size scales (ROSS) introduced in Section 2.3, which is measured by $h$. The patches that contribute to different ROSS have to be separated by strong localized barriers, otherwise the rupture can easily jump the barriers. This is the case for fractal heterogeneities with small standard deviation. Therefore, model $F$ requires two parameters to tune the fault towards NROSS or WROSS, as discussed in Section 2.3. Model RW needs only one parameter to control the ROSS, and it is probably also closer to natural faults due to the existence of near-vertical fault offsets. We conclude that model RW gives a better performance of both the ability to tune effectively the level of fault heterogeneities and the relation between model simulations and natural seismicity.

The results of the simulations with heterogeneous faults can be summarized in a phase diagram. According to the results of Figures 4-7, the parameter $h$ represents a third dimension in addition to the stress loss $\gamma$ and the dynamic weakening $\varepsilon$. Figure 8(a) shows a projection of the 3-D phase diagram onto the plane $\gamma=0$ and Figure 8(b) gives a similar projection for $\varepsilon=$ constant. As discussed by BEN-ZION et al. (1999), DAHMEN et al. (1998), and Fisher et al. (1997), cases with $\varepsilon=0$ and $\gamma=0$ represent a critical point of a phase transition in the 2-D phase diagram. To illustrate the difference between purely static friction $(\varepsilon=0)$ and the static-kinetic friction $(\varepsilon>0$ ), two frequency-size distributions are shown in Figure 9, one for $\varepsilon=0$ (for all cells) and another for $\langle\varepsilon\rangle=0.02$. Both are calculated on a fault with heterogeneities according to Figure 2(a). As discussed in Section 2.2, a heterogeneous fault includes space-dependent dynamic weakening coefficients $\varepsilon$, because of varying stress drops $\Delta \tau$ and constant dynamic overshoot DOS. Therefore, the value of $\varepsilon$ in the phase diagram (Fig. 8) should be understood as a mean value. The frequency-size distribution for $\varepsilon=0$ follows a Gutenberg-Richter behavior tapered by finite-size 
effects, while $\varepsilon>0$ leads to a characteristic earthquake distribution. The precise structure of the phase diagrams of Figure 8 should be clarified in a future work.

\subsection{Investigation of Rupture Histories}

In contrast to the earlier quasi-static version of the model (e.g., BEN-ZION, 1996), the present quasi-dynamic model simulates the spatio-temporal evolution of stress and slip with a finite propagation velocity $v_{s}$. This allows us to study general properties of rupture histories for single earthquakes, in particular events which rupture large parts of the fault. It can be expected that quenched heterogeneities significantly influence the stress propagation, especially if high stress drop barriers are included. As pointed out in Section 2.1, the creep mechanism already creates some quenched spatial heterogeneities, because the mixture of brittle and ductile processes produces heterogeneous effective rheology. Therefore, we compare rupture histories of two extreme cases in this section: (1) A fault without creep motion and uniformly distributed brittle properties: $\tau_{s}=10 \mathrm{MPa}, \Delta \tau=1 \mathrm{MPa}+$ ran $\cdot 8 \mathrm{MPa}$, where ran is a uniformly distributed random number between 0 and 1. (2) A fault with creep motion and heterogeneous brittle properties according to Figure 2(b). Both simulations start with randomly distributed initial stresses $\tau \in\left[\tau_{a} ; \tau_{s}\right)$.

Figures 10 and 11 show snapshots from rupture histories of mainshocks in both models described above. While Figure 10 corresponds to a system-wide event with area $A=4096$ cells, the rupture area in Figure 11 is smaller having $A=2093$ cells. Both events were recorded after the model had evolved for some 1000's of years. The gray scaled boxes show the dimensionless stress $\hat{\tau}=\left(\tau-\tau_{a}\right) /\left(\tau_{s}-\tau_{a}\right)$, which is 1 for
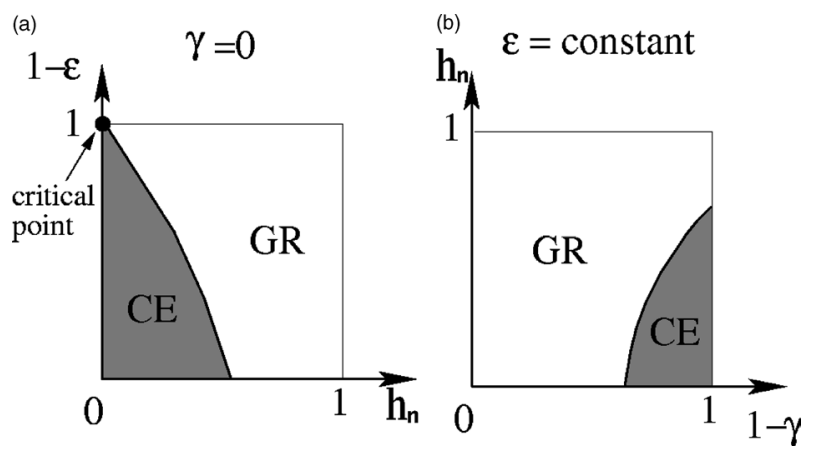

Figure 8

Projections of the 3-D schematic phase diagrams for $\gamma=0$ (panel (a)) and $\varepsilon=$ constant (panel (b)). The value $h_{n}=h / h_{\max }$ denotes the heterogeneity parameter normalized to the interval $[0 ; 1]$. CE denotes the characteristic earthquake distribution and GR the Gutenberg-Richter distribution. Note that $\varepsilon$ represents the mean value of the dynamic weakening coefficient over the fault; due to a constant dynamic overshoot $D O S=1.25$ and space-dependent stress drops (see Figs. 2 and 3), $\varepsilon$ is also space-dependent. The finite-size effects at $h_{n} \approx 1$ (see text) are not taken into account here; that is, the phase diagram is assumed to represent a fault of infinite size. 


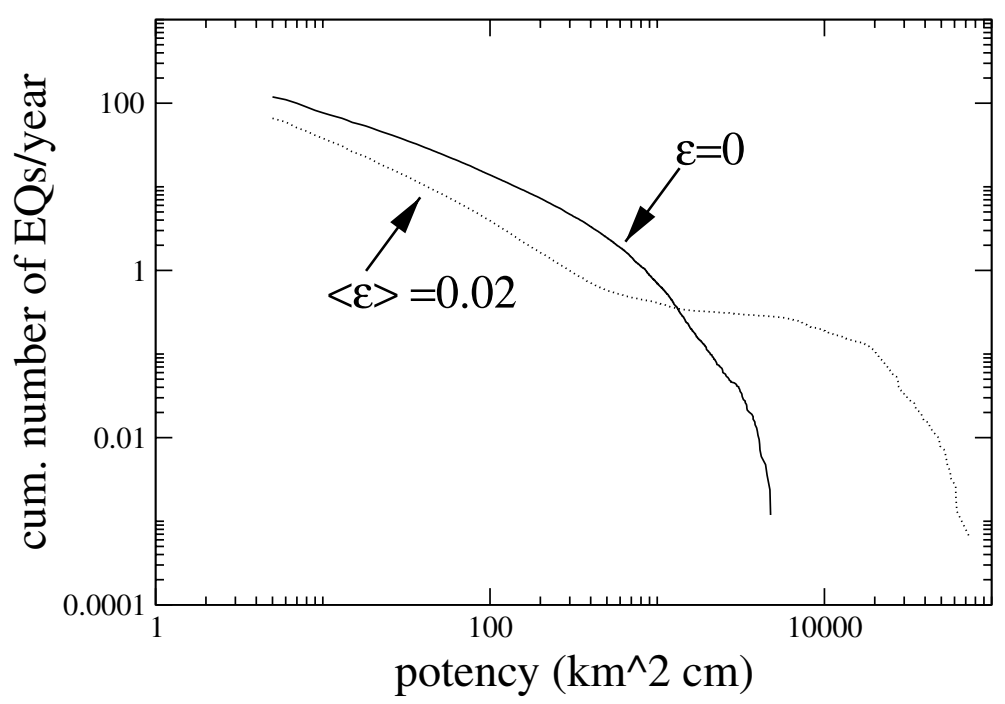

Figure 9

Comparison of frequency-size distributions for a model at the critical point $C$ ( $\gamma=\varepsilon=0$, solid line) and a noncritical model realization with finite $\varepsilon$ (dashed line). Note that the presence of heterogeneities (here according to Figure 2(a)) leads to space-dependent dynamic weakening.

a critical cell (light color) and 0 for a the lowest stress value $\tau_{a}$ (dark color). It is remarkable that the situation on the homogeneous fault before the occurrence of a large earthquake has evolved to clearly defined patches with highly loaded boundaries (Fig. 10). The mainshock is initiated at a boundary of a patch and propagates from the boundary inward. After the system-wide event, the plate motion reloads the fault and the stress evolves to form new similar spatial patterns. In an intermediate size earthquake, not all patches are ruptured. This behavior is similar to a rupture scenario of "double encircling pincers," which have been found by DAS and Kostrov (1983) in a dynamic fault model with a circular asperity. However, DAS and Kostrov (1983) observe an almost isotropic propagation inward, while the patches in our model are mostly ruptured from a preferred direction.

A completely different scenario is observed in the highly heterogeneous model with creep motion. The stress field before a mainshock has no obvious regularity, except the brittle-ductile transition (here at about $15 \mathrm{~km}$ depth), which is responsible for the stable sliding of the deep cells. After the first large self-organized patch is ruptured from the hypocenter in Figures 11(a) and 11(b), a barrier at about $15 \mathrm{~km}$ along strike is jumped and the next patch is ruptured. Due to the irregular stress field and long-range interaction, different regions of the fault can be ruptured simultaneously, e.g., in Figure 11(c) a new patch at the right boundary of the fault is created while the rupture adjacent to the hypocenter continues to evolve. This is in contrast to the smooth fault, where most of the patches are ruptured almost sequentially. The 

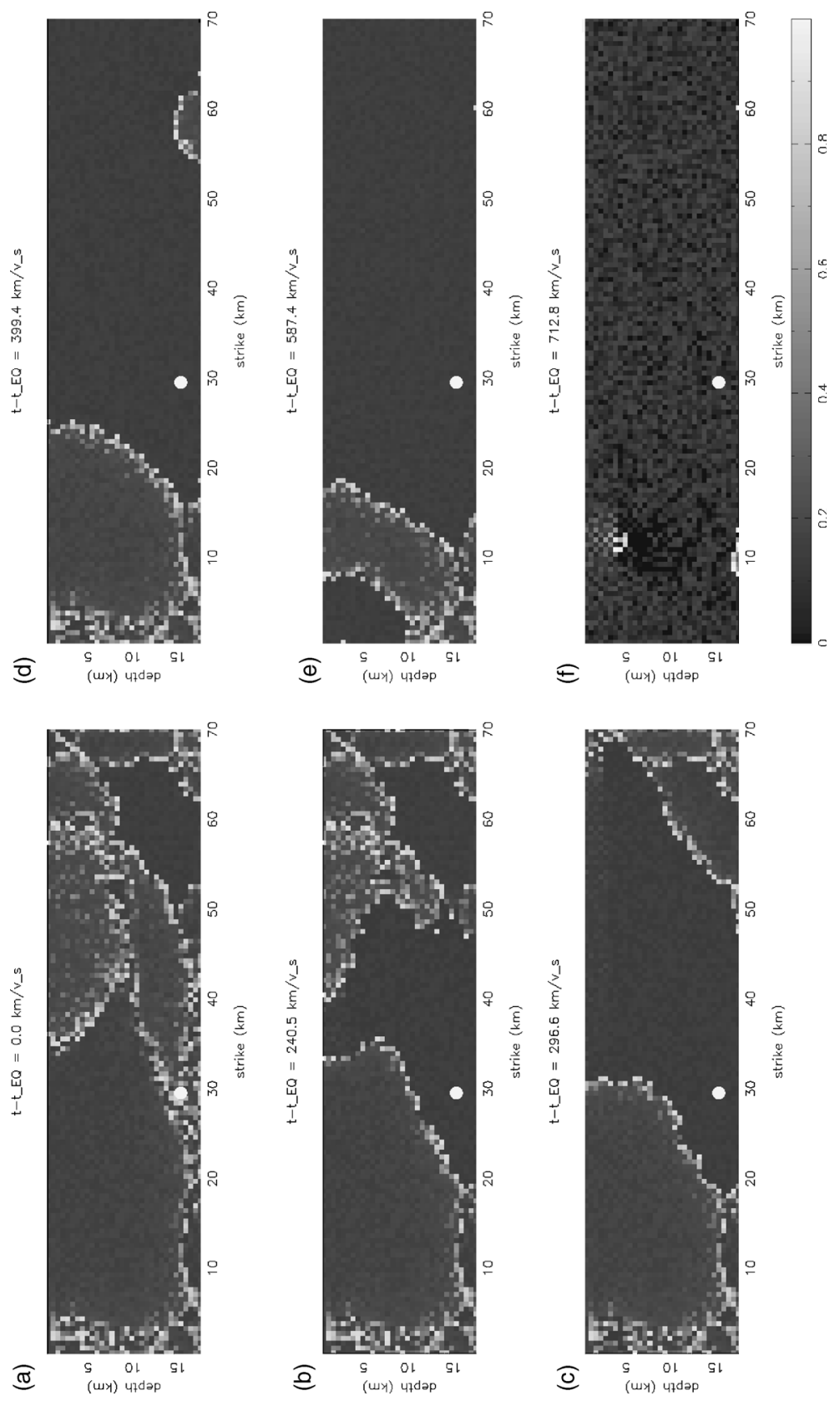

Figure 10

Snapshots of rupture evolution for a system-wide event on a smooth fault without creep motion. $t-t_{E Q}$ denotes the time after the rupture initiation and depends on the choice of the shear wave velocity $v_{s}$. The filled white circle is the hypocenter of the event. The figure shows the dimensionless stress state $\hat{\tau}=\frac{\tau-\tau_{a}}{\tau_{s}-\tau_{a}}$ of the cells. 

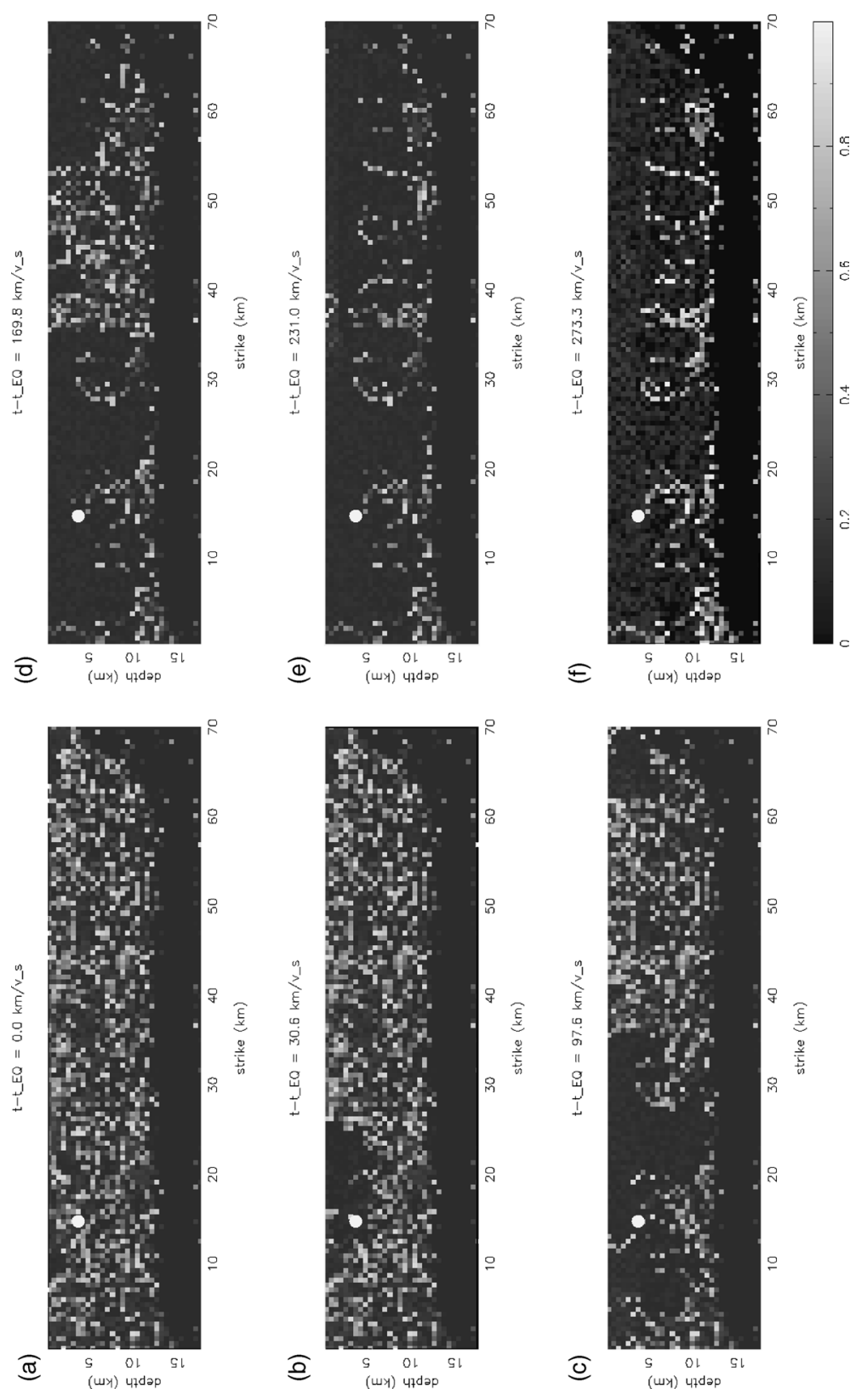

Figure 11

Same as Figure 10 for a fault with heterogeneities according to 2(b) with a brittle-ductile transition at about $15 \mathrm{~km}$ depth. 
overall rupture propagation on the heterogeneous fault is relatively slow, because some cells undergo multiple slips due to the heterogeneous distribution of stress drops and slow the rupture process. During the rupture and especially at the end, quasi-vertical stress patterns are created from time to time, e.g. in Figure 11(e) and 11(f). These patterns reflect the high stress drop barriers in Figure 2(b). At the end of the mainshock, the fault is almost unloaded between these near-vertical barriers. A highly irregular rupture history similar in some respects to the results of Figure 11, was observed during the Chi-Chi (Taiwan) earthquake on September 21, 1999 $\left(M_{w}=7.6\right)$ (SHIN and TENG, 2001).

Finally, we show in Figure 12 three snapshots of a model at the critical point $\varepsilon=0, \gamma=0$ (see Fig. 8(a)). The snapshots were taken before, during and after a large event with area $A=2199$ cells. The only difference from the smooth model leading to Figure 10 is the choice of a purely static friction $\left(\tau_{d}=\tau_{s}\right)$ instead of static-kinetic friction. Despite the high degree of smoothness of brittle parameters, the stress field has no obvious structure and the rupture consists of a sequence of almost uncorrelated cell slips. This is in agreement with the analytical results of FISHER
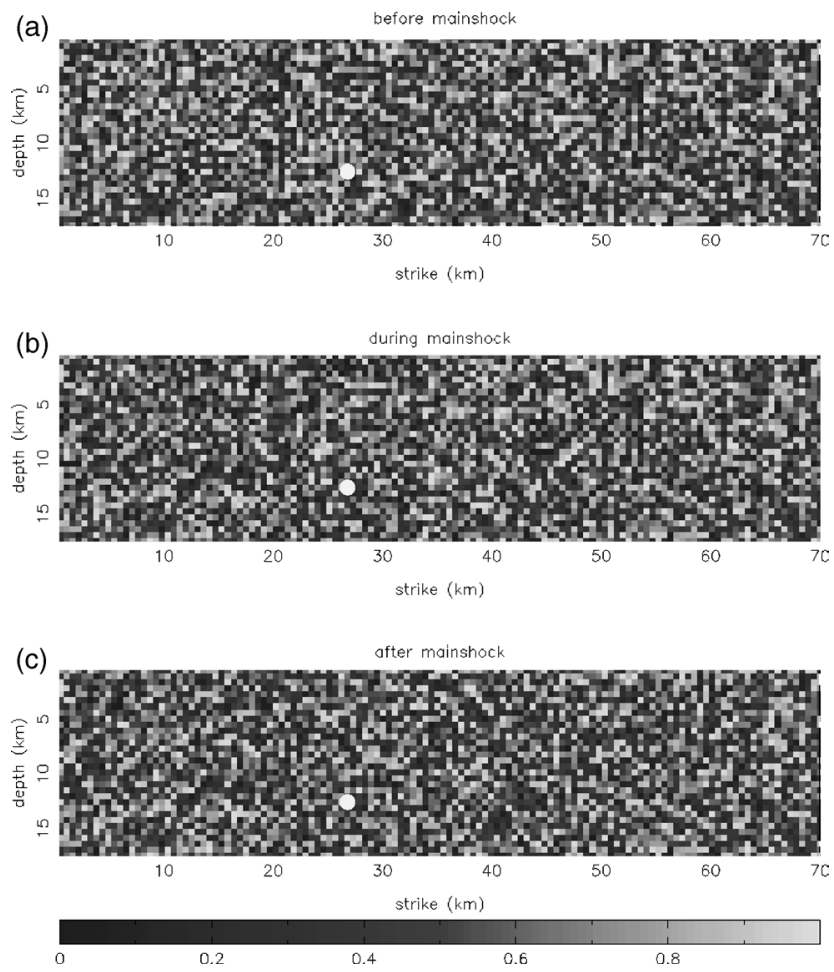

Figure 12

Same as Figure 10 for purely static friction $\left(\tau_{s}=\tau_{d}\right)$ corresponding to the critical point in Figure 8(a): normalized stress field before (panel (a)), during (panel (b)), and after (panel (c)) a mainshock with area $A=2199$ cells). 
et al. (1997) and similar in some respects to the simulations of Figure 11. As shown in Figure 9, the frequency-size statistics for $\varepsilon=0, \gamma=0$ follow the Gutenberg-Richter law without the enhanced occurrence of large events.

\section{Discussion and Conclusions}

We have investigated the effect of quenched spatial heterogeneities in a quasidynamic version of a discrete strike-slip fault in a 3-D elastic half space (BENZION, 1996, BEN-ZION and RICE, 1993; ZÖLler et al., 2004). The calculations employed two types of heterogeneities: 1 . near-vertical barriers of high brittle stress drop embedded in a fault with low brittle stress drop (RW) and 2. power-law stress drop distributions $(\mathrm{F})$. The former type probably provides a closer representation of natural faults because it simulates fault offsets which are common geological features (Wesnousky 1994; Ben-Zion and SAmmis, 2003). Both types of heterogeneities can be tuned with respect to the degree of spatial complexity between the two end-member cases of a smooth fault (few barriers/low fractal dimension) and a rough fault (many barriers/high fractal dimension). However, large variations in the parameters of model $\mathrm{F}$ produce a small effect on the obtained earthquake behavior.

The results indicate that the degree of spatial disorder can act as a tuning parameter for the earthquake dynamics. Smooth faults are governed by characteristic earthquake behavior, connected slip areas and cyclic components in various time histories like potency release. In contrast, seismicity on rough faults is characterized by a Gutenberg-Richter law, disconnected slip areas and fractal-like time histories. FISHER et al. (1997), DAHMEN et al. (1998), and BEN-ZION et al. (2003) showed with related quasi-static models that similar differences exist on these and other earthquake quantities as the dynamic weakening parameter $\varepsilon$ moves toward or away from a critical values $(\varepsilon=0)$.

Although $h$ may act as a tuning parameter for the heterogeneities, the results suggest that the basic underlying physical quantity is the range of size scales (ROSS) that characterizes the heterogeneities. This is clear in the case of the RW heterogeneities. If an earthquake is initiated in a cell with a low brittle stress drop, the rupture can propagate easily until a high stress drop barrier is reached. Then the barrier can overcome the stress concentration at the rupture front with some probability. On the other hand, if an earthquake starts in one of the barriers, it is likely that the adjacent low stress drop regions will be ruptured, resulting in a large event. Therefore, the low and intermediate events will organize in the patches between the barriers. Consequently, a large range of size scales of such patches will result in a Gutenberg-Richter law, and a narrow range will favor characteristic events. Thus the ROSS of the heterogeneities is the key property controlling the frequency-size distribution as suggested by BEN-ZION and RICE (1995), BEN-ZION 
(1996), and BEN-ZION et al. (2003). A narrow range of size scales (NROSS) leads to characteristic earthquake behavior, while a wide range of size scales (WROSS) results in a Gutenberg-Richter distribution.

This behavior is also somewhat valid in a weaker form for the power-law stress drop distribution, where in this case the fault is overall smoother due to the continuous distribution of stress drops in contrast to the sharp bimodal distribution of the RW heterogeneities. The comparison of the two types of heterogeneities unveils the relevance of an additional tuning parameter in model $\mathrm{F}$, the standard deviation of the stress drops. If this range of values is small, a rupture can easily jump heterogeneities, although the degree of spatial disorder may be high. This occurs for power-law distributions of the stress drop due to the continuous range of values. Therefore power-law distributions require two tuning parameters, the fractal dimension and the standard deviation in order to tune the heterogeneities between NROSS and WROSS. The RW model needs only the tuning parameter $h$ for this aim, because the almost discontinuous shape of the barriers defines the patches where ruptures can propagate.

A qualitative analysis of rupture histories of mainshocks in the case of faults with low and high degrees of disorder reveals clear differences in the spatio-temporal evolution of stress. On smooth faults, the stress field organizes itself into large patches with highly loaded boundaries. A mainshock starts at one of the boundaries and ruptures a patch in the inward direction. For a system-wide event all patches are ruptured more-or-less in series. This highly organized pattern formation vanishes if the static-kinetic friction is replaced by a purely static friction $\left(\tau_{d}=\tau_{s}\right)$. Assuming full stress conservation during rupture $(\gamma=0)$, this case represents a critical point in the phase diagram Figure 8(a), where no system-wide event occurs. If dynamic weakening is switched on $(\varepsilon>0)$, earthquakes bigger than a certain size become unstoppable (BEN-ZION and Rice, 1993; DAHMEN et al., 1998), unless the fault heterogeneities have a WROSS. In the latter case, the stress field before a mainshock is highly irregular also for a finite dynamic weakening $(\varepsilon>0)$ without large selforganized patches. The rupture propagates simultaneously in several regions and is slowed down or terminated if a barrier is reached.

A shortcoming of our simulations that should be addressed in future studies is the effect of finite size of the fault, which limits the range of $h$ values. A more efficient design of the computer code will provide a possibility perform parameter space studies on a larger fault and quantify more clearly the role of the heterogeneities on earthquake dynamics. The results of this paper indicate that heterogeneities have dramatic effects on the spatio-temporal evolution of rupture histories and earthquake statistics. These effects should be clarified further with more detailed analyses, e.g., by using stress and seismicity functions of the type employed by Ben-Zion et al. (2003). Another interesting problem for future studies is a joint theoretical-observational work involving tuning the model parameters to specific fault zones (e.g., the Parkfield section of the San Andreas fault). Large 
statistically-complete data sets of simulated slip histories and events statistics for various cases of such tuned models may provide important input for models of seismic hazard assessment.

\section{Acknowledgments}

This work was supported by the collaborative research center "Complex Nonlinear Processes" (SFB555) of the "German Research Society" (DFG). YBZ acknowledges support from a Mercator fellowship of the DFG. The manuscript benefited from comments by two anonymous referees and Ramón Zúñiga.

\section{REFERENCES}

BEn-ZIon, Y. (1996), Stress, slip, and Earthquakes in Models of Complex Single-fault Systems Incorporating Brittle and Creep Deformations, J. Geophys. Res. 101, 5677-5706.

Ben-Zion, Y. (2001), Dynamic Rupture in Recent Models of Earthquake Faults, J. Mech. Phys. Solids 49 , 2209-2244.

BEN-Zion, Y. (2003), Key Formulas in Earthquake Seismology, International Handbook of Earthquake and Engineering Seismology, Part B, 1857-1875.

Ben-Zion, Y., Dahmen, K., Lyakhovsky, V., Ertas, D., and Agnon, A. (1999), Self-driven Mode Switching of earthquake activity on a fault system, Earth and Plan. Sci. Lett., 172, 11-21.

Ben-Zion, Y., M. Eneva, and LiU, Y. (2003), Large Earthquake Cycles and Intermittent Criticality on Heterogeneous Faults due to Evolving Stress and Seismicity, J. Geophys. Res. 108, doi 1029.2002JB002121.

BEN-ZION, Y. and RiCE, J. R. (1993), Earthquake Failure Sequences along a Cellular Fault Zone in a Threedimensional Elastic Solid Containing Asperity and Nonasperity Regions, J. Geophys. Res. 98, 14,10914,131 .

Ben-Zion, Y. and Rice, J. R. (1995), Slip Patterns and Earthquake Populations along Different Classes of Faults in Elastic Solids, J. Geophys. Res. 100, 12,959-12,983.

Ben-Zion, Y. and Rice, J. R. (1997), Dynamic Simulations of Slip on a Smooth Fault in an Elastic Solid, J. Geophys. Res. 102, 17,771-17,784.

Ben-Zion, Y. and SAmmis, C. G. (2003), Characterization of fault zones, Pure Appl. Geophys, 160, 677-715. Binney, J. J., Dowrick, N. J., Fisher, A. J., and Newman, M. E. J. The Theory of Critical Phenomena (Oxford University Press 1993).

Chinnery, M. (1963), The Stress Changes that Accompany Strike-slip Faulting, Bull. Seismol. Soc. Am. 53 , 921-932.

DAhmen, K., ERtAs, D., and Ben-ZiOn, R. (1998), Gutenberg-Richter and Characteristic Earthquake Behavior in Simple Mean-field Models of Heterogeneous Faults, Phys. Rev. E 58, 1494-1501.

Das, S. and Kostrov, B. V. (1983), Breaking of a Single Asperity-rupture Process and Seismic Radiation, J. Geophys. Res. 88, 4277-4288.

Fisher, D. S., Dahmen, K., Ramanathan, S., and Ben-Zion, Y. (1997), Statistics of Earthquakes in Simple Models of Heterogeneous Faults, Phys. Rev. Lett. 78, 4885-4888.

Gutenberg, G. and Richter, C. F. (1956), Earthquake Magnitude, Intensity, Energy and Acceleration, Bull. Seismol. Soc. Am. 46, 105-145.

Hainzl, S. and Zöller, G. (2001), The Role of Disorder and Stress Concentration in Nonconservative Fault Systems, Physica A 294, 67-84.

Hainzl, S., Zöller, G., and Kurths, J. (1999), Similar Power Laws for Fore- and Aftershock Sequences in a Spring-block Model for Earthquakes, J. Geophys. Res. 104, 7243-7253. 
Heimpel, M. H. (2003), Characteristic Scales of Earthquake Rupture from Numerical Models, Nonlin. Proc. Geophys. 10, 573-584.

Lomnitz-Adler, J. (1999), Automaton Models of Seismic Fracture: Constraints Imposed by the Magnitudefrequency Relation, J. Geophys. Res. 98, 17,745-17,756.

Madariaga, R. (1976), Dynamics of an Expanding Circular Fault, Bull. Seismol. Soc. Am. $66639-666$.

Mehta, A. P., DAhmen, K. A., and Ben-Zion, Y. (2005), From Magnets to Earthquakes: Universal Mean Moment Rate Profiles of Earthquake Ruptures, submitted to Phys. Rev. Lett.

Robinson, R., and Benites, R. (2001), Upgrading a Synthetic Seismicity Model for More Realistic Fault Ruptures, Geophys. Res. Lett. 28, 1843-1846.

Shin T.C., Teng T.L. (2001), An Overview of the 1999 Chi-Chi, Taiwan, Earthquake, Bull. Seismol. Soc. Am. 91, 895-913.

SteAcy, S. J. and Mccloskey, J. (1999), Heterogeneity and the Earthquake Magnitude-frequency Distribution, Geophys. Res. Lett. 26, 899-902.

Stirling, M. W., Wesnousky, S. G., and Shimazaki, K. (1996), Fault Trace Complexity, Cumulative Slip, and the Shape of the Magnitude-frequency Distribution for Strike-slip Faults: A Global Survey, Geophys. J. Int. 126, 301-301.

Turcotte, D. L. Fractals and Chaos in Geology and geophysics (Cambridge University Press, New York 1997).

Utsu, T. (2002), Statistical Features of Seismicity, International Handbook of Earthquake and Engineering Seismology, Part B, 719-732.

Weatherley, D., Mora, P., and XiA, M. F. (2002), Long-range Automaton Models of Earthquakes: Power-law Accelerations, Correlation Evolution, and Mode-switching, Pure Appl. Geophys. 159, 24692490.

Weasnousky, S. G. (1994), The Gutenberg-Richter or Characteristic Earthquake Distribution, which is it?, Bull. Seismol. Soc. Am. 84, 1940-1959.

Wilson, K. G. (1979), Problems in Physics with Many Scales of Length, Scientific American 241, 158-179.

Wyss, M., Schorlemmer, D., and Wiemer, S. (2000), Mapping Asperities by Minima of Local Recurrence Time: San Jacinto-Elsinore Fault Zones, J. Geophys. Res. 105, 7829-7844.

Zöller, G., Holschneider, M., and Ben-ZIon, Y. (2004), Quasi-static and Quasi-dynamic Modeling of Earthquake Failure at Intermediate Scales, Pure Appl. Geophys. 161, 2103-2118.

(Received December 12, 2003, accepted August 14, 2004)

(10) To access this journal online:
http://www.birkhauser.ch 